\title{
Review
}

Medical Principles

and Practice

\section{Targeted Therapy with Biologicals and Small Molecules in Primary Immunodeficiencies}

\author{
Ottavia Maria Delmonte Luigi Daniele Notarangelo \\ Laboratory of Clinical Immunology and Microbiology, Division of Intramural Research, National Institute of Allergy \\ and Infectious Diseases, National Institutes of Health, Bethesda, MD, USA
}

\section{Significance of the Study}

- Several primary immune deficiencies are associated with immune dysregulation and hyperinflammation.

- A growing understanding of the pathophysiology of these disorders has led to the development of mechanism-based therapeutic strategies.

- Small molecules and biologics are effective in reversing clinical manifestations of primary immune deficiencies and as a bridge treatment to hematopoietic stem cell transplantation.

\section{Keywords}

Primary immunodeficiency diseases .

Immunodysregulation - Combined immunodeficiency .

Precision medicine $\cdot$ Monoclonal antibody · Small molecule inhibitor

\section{Abstract}

Primary immunodeficiencies are disorders resulting from mutations in genes involved in immune defense and immune regulation. These conditions are characterized by various combinations of recurrent infections, autoimmunity, lymphoproliferation, inflammatory manifestations, and malignancy. In the last 20 years, newborn screening programs and next generation sequencing techniques have increased the ability to diagnose primary immunodeficiencies. Furthermore, an advanced understanding of the molecular ba- sis of these inherited disorders has led to the implementation of targeted therapies that utilize small molecules and biologics to modulate the activity of impaired intracellular pathways. This article will discuss selected primary immunodeficiencies, the genetic defects of which have been recently studied and are amenable to targeted therapy as a reflection of the potential of precision medicine in the future.

(C) 2019 The Author(s)

Published by S. Karger AG, Basel

\section{Introduction}

The broad availability of whole exome and whole genome sequencing analysis has made possible the discovery of an increasing number of genetic disorders of the immune system over the last 2 decades. The International Union of Immunological Societies has updated the classi-

\begin{tabular}{ll}
\hline KARGER & @ 2019 The Author(s) \\
& Published by S. Karger AG, Basel Oparger \\
karger@karger.com & This article is licensed under the Creative Commons Attribution- \\
www.karger.com/mpp & NonCommercial-NoDerivatives 4.0 International License (CC BY- \\
& NC-ND) (http://www.karger.com/Services/OpenAccessLicense). \\
& Usage and distribution for commercial purposes as well as any dis- \\
tribution of modified material requires written permission.
\end{tabular}


Table 1. Targeted therapies used in PID

\begin{tabular}{|c|c|c|c|}
\hline Molecular structure & Molecular target & Drug & Indication \\
\hline Macrolide compound & mTOR & Sirolimus & $\begin{array}{l}\text { NLCR4-GOF } \\
\text { POMP deficiency } \\
\text { CTLA-4 haploinsufficiency } \\
\text { APDS }\end{array}$ \\
\hline CTLA-4 IgG fusion protein & B7-1 (CD80), B7-2 (CD86) & $\begin{array}{l}\text { Abatacept } \\
\text { Belatacept }\end{array}$ & $\begin{array}{l}\text { CTLA-4 haploinsufficiency LRBA deficiency } \\
\text { CTLA-4 haploinsufficiency }\end{array}$ \\
\hline $\begin{array}{l}\text { Antihuman IL-1 IgG1 mAb } \\
\text { IgG1 linked to IL-1R and IL-1R accessory protein }\end{array}$ & IL- $1 \beta$ & $\begin{array}{l}\text { Canakinumab } \\
\text { Rilonacept }\end{array}$ & $\begin{array}{l}\text { CAPS } \\
\text { FCAS } \\
\text { MWS } \\
\text { DIRA }\end{array}$ \\
\hline IgGlk recombinant humanized $\mathrm{mAb}$ & IL-6R & Tocilizumab & STAT3-GOF \\
\hline Small molecule inhibitor & $\begin{array}{l}\text { JAK1 and JAK } 2 \\
\text { JAK } 1 \text { and JAK3 } \\
\text { P110 }\end{array}$ & $\begin{array}{l}\text { Ruxolitinib } \\
\text { Baricitinib } \\
\text { Tofacitinib } \\
\text { Leniolisib }\end{array}$ & $\begin{array}{l}\text { STAT3-GOF } \\
\text { STAT1-GOF } \\
\text { CANDLE syndrome } \\
\text { APDS }\end{array}$ \\
\hline Recombinant IL-18 binding protein & IL-18-binding protein & Tadekinig- $\alpha$ & NLCR4-GOF \\
\hline
\end{tabular}

fication of genetic defects associated with primary immune deficiencies (PID) to more than 350 disorders. Many of the newly discovered PID manifest with a broad phenotypical spectrum to include not only variable degrees of susceptibility to infections but also immune dysregulation manifesting as autoimmunity and/or hyperinflammation. The understanding of the immunobiology underpinning the pathophysiology of immune dysregulation has evolved significantly. Molecular studies have unraveled signaling pathways and metabolic requirements that are crucial to tightly control immune and inflammatory responses. As such, the capacity to precisely identify the molecular basis of immunological disorders has also largely increased, paving the path for the application of targeted therapeutic agents that replace, or can be combined with, traditional immunosuppressant treatments. These newly generated drugs aim to modulate the activity of intracellular pathways whose function is either increased or decreased as a result of a specific genetic defect. In this review, we illustrate various recently described PID, focusing in particular on selected disorders associated with immune dysregulation and hyperinflammation. We also present targeted therapeutic approaches based on the molecular mechanisms underpinning these diseases (Table 1).

\section{Precision Medicine in Immune Dysregulation Disorders}

Activated Phosphoinositide 3-Kinase $\delta$ Syndrome

Activated phosphoinositide 3-kinase $\delta$ syndromes 1 and 2 (APDS1 and APDS2) are combined immunodeficiency (CID) disorders due to mutations in either the PIK3CD or the PIK3R1 gene [1-3]. These genes encode for the $\mathrm{p} 110 \delta$ catalytic subunit and the $\mathrm{p} 85 \mathrm{a}$ regulatory subunit of phosphoinositide 3-kinase (PI3K), respectively. PI3K phosphorylates phosphatidylinositol-4,5 biphosphate (PIP2) to phosphatidylinositol-3,4,5 trisphosphate (PIP3), an important mediator of PI3K downstream cellular pathways including mammalian target of rapamycin (mTOR).

In 2014 Lucas et al. [1] and Angulo et. al. [2] identified heterozygous gain-of-function (GOF) mutations in PIK3CD in patients with a CID phenotype (APDS1) [1, $2]$. Soon thereafter it was reported that heterozygous lossof-function (LOF) mutations in PIK3R1 result in a similar clinical phenotype (APDS2) [3]. Since then, different heterozygous mutations have been reported. However, the E1021K amino acid substitution is by far the most common in APDS1, while in APDS2 a heterozygous donor 
splice site mutation causing skipping of exon 11 is the main disease-causing alteration [3-5]. The consequent loss of the p $110 \delta$-binding site in exon 11 results in the loss

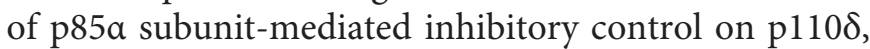
thus causing hyperactivation of the PI3K pathway [3]. The clinical phenotypes of APDS1 and APDS2 significantly overlap $[6,7]$. Both diseases are characterized by the coexistence of immunodeficiency with a high susceptibility to infections and autoimmunity, lymphoproliferation, and an increased risk of lymphoma. The onset of the disease is typically in childhood, with sinopulmonary infections often leading to bronchiectasis over time. Respiratory infections are mainly due to Streptococcus pneumoniae and Haemophilus influenza [6]. Recurrent or persistent infections due to Herpesviridae, such as Epstein-Barr virus, cytomegalovirus, herpes simplex virus, and Varicella-Zoster virus, are also frequent [6]. Lymphadenopathy, splenomegaly, and/or hepatomegaly represent the clinical signs of lymphoproliferation and are present in the majority of the patients. Other clinical characteristics include autoimmunity, lymphoid hyperplasia of the airways and gut, developmental delay, and enteropathy [8]. Moreover, APDS patients are at a higher risk for lymphomas (particularly Epstein-Barr virus-driven $B$ cell lymphoma) $[6,7]$.

Growth retardation has been reported in approximately $50 \%$ of APDS 2 patients, but not in APDS1; this may be explained by the dysregulated activity of the $\mathrm{p} 110 \mathrm{a}$ and $110 \beta$ PI3K subunits $[4,5,7]$. The immunological phenotype of APDS includes both T-cell abnormalities with decreased naive $\mathrm{T}$ cells, increased $\mathrm{T}$ effector memory cells, and exhausted $\mathrm{T}$ effector memory reexpressing CD45RA ( $\mathrm{T}_{\text {EMRA }}$ ) cells and high numbers of $\mathrm{T}$ follicular helper (Tfh) cells. B-cell impairment is also present as indicated by variable degrees of hypogammaglobulinemia, elevated IgM levels and high numbers of transitional B cells, decreased switched memory cells, and an impaired response to vaccinations [1]. Increased AKT and S6 phosphorylation in T and B cells have been observed in APDS patients as a result of augmented mTOR signaling [1], thereby supporting the use of mTOR-targeted therapy to control the disease [9]. Standard treatments for APDS include antimicrobial prophylaxis and immunoglobulin replacement to prevent infectious complications [7]. Different combinations of immune suppressive regimens have been used to control lymphoproliferation and autoimmunity, with the best results being obtained with rituximab and mTOR inhibitors (such as rapamycin) [9]. Hematopoietic stem cell transplantation (HSCT) has been successful in reversing the clinical phenotype especially in

Mechanism-Based Treatment of Primary Immunodeficiencies the context of optimal chimerism; however, elevated rates of post-transplant viral reactivation and engraftment failure at different time points have been reported $[10,11]$.

Selective PI3K $\delta$ inhibitors represent a targeted therapy based on characterization of the molecular mechanisms underpinning APDS. Two phase 2 trials are currently ongoing to establish the safety and efficacy of these drugs. The first trial is based on oral administration of leniolisib (NCT02435173), while the second one is based on inhaled nemiralisib (NCT02593539). The initial results of the first trial, with dose-escalating administration of leniolisib for 12 weeks, showed that the drug was well tolerated; reduction of lymphadenopathy and splenomegaly and improvement of cytopenias were reported following the therapy [12].

However, despite the promising results of these targeted therapy, in patients with treatment-refractory disease and those with drug-related adverse events, HSCT represent an option that must be taken into account. In addition, the long-term safety profile of $\mathrm{MTOR}$ and PI3K $\delta$ inhibitors in patients with APDS has yet to be fully defined, especially considering some evidence that $\mathrm{P} 110 \delta$ inhibitors may lead to genomic instability in B cells [13].

\section{CTLA4 Haploinsufficiency}

Cytotoxic lymphocyte antigen-4 (CTLA4) haploinsufficiency is due to heterozygous germline mutations in the CTLA4 gene. CTLA4 (also defined as CD152) is a receptor expressed by $\mathrm{T}$ cells that inhibits cell activation and immune response. CTLA 4 binds to 2 different ligands on antigen presenting cells CD80 and CD86 [14]. Upon ligand binding, CTLA4 produces an inhibitory signal that limits the activation and proliferation of $\mathrm{T}$ cells. CD28, a T-cell costimulatory molecule, also binds to CD80/86 with an opposite effect [14]. CTLA4 expression is crucial for $\mathrm{T}$ regulatory cell function and immune tolerance as well. Two groups originally reported the presence of CTLA4 mutations in patients with recurrent sinopulmonary and viral infections, associated with autoimmunity and lymphoproliferation $[15,16]$. Clinical and laboratory findings were consistent with common variable immunodeficiency, but patients also suffered from T-cell infiltrates in the lungs, the gastrointestinal tract, and the nervous system, as well as significant autoimmune blood cytopenia. The disease is characterized by incomplete penetrance and variable expressivity $[15,16]$. Functional studies showed diminished expression of CTLA4 associated with impaired suppressor function in FOXP3+ Treg cells [16]. Moreover, patients had a decreased expression 
of CTLA 4 on the surface of activated conventional T cells, suggesting that impaired expression of this molecule may cause a defective capacity to extinguish $\mathrm{T}$-cell responses and to control self-reactive T cells that have escaped central deletion. Also the $\mathrm{B}$ cell compartment is defective in these patients, with a progressive loss of $\mathrm{B}$ cells and an increased proportion of autoreactive CD2 $1^{\text {low }} \mathrm{B}$ cells [15]. Recently, Schwab et al. [17] reported on a cohort of 133 patients with CTLA4, broadening the clinical and immunological spectrum associated with this disease. Clinical manifestations of this series included severe or refractory autoimmune blood cytopenias, nonmalignant lymphoproliferation, and respiratory and gastrointestinal disease. Respiratory tract findings included recurrent lower and upper respiratory tract infections, lymphocytic interstitial lung disease, bronchiectasis, and lung fibrosis. Gastrointestinal manifestations were present, with enteropathy and Crohn's-like colitis often being particularly severe. The immunological phenotype included CD4 T-cell lymphopenia, variable degrees of hypogammaglobulinemia, and impaired response to immunizations and Bcell maturational defects [17].

Identification of CTLA4 as a key immune regulator has led to the generation of abatacept and belatacept molecules derived from the fusion between the extracellular domain of CTLA4 and the Fc region of IgG1, functioning in vivo as inhibitors of T-cell activation [18]. Side effects of these drugs are related to their immune suppressive activity and include increased susceptibility to infections (especially viral) and to malignancy.

Initially, rapamycin was used as immunosuppressive therapy in CTLA4 haploinsufficiency; however, abatacept and belatacept have been shown to be an effective targeted treatment to control the immune dysregulation seen in this disorder [18].

Lee et al. [19] reported on a 14-year-old girl affected by CTL4 haploinsufficiency with severe enteropathy, autoimmune hepatitis, and autoimmune blood cytopenia. Initiation of abatacept therapy diminished the diarrhea, controlled the autoimmune hemolytic anemia, and eliminated the need for additional immunosuppressive agents. In the cohort described by Schwab et al. [17], 11 patients received abatacept or belatacept and experienced improvement of autoimmune manifestations, lymphadenopathy, and lymphoproliferation in the lungs. Sirolimus was used in 13 patients with a good response in terms of reduced cytopenia, splenomegaly and lymphadenopathy [17].

Interestingly, another target therapy, i.e., vedolizum$\mathrm{ab}$, a humanized monoclonal antibody ( $\mathrm{mAb}$ ) that targets
T cells expressing the $\alpha 4 \beta 7$ integrin gut homing receptor, was successfully used to treat a CTLA4 patient with refractory autoimmune enterocolitis [20]. However, as expected, vedolizumab did not improve the hypogammaglobulinemia and pure red cell aplasia in the patient [20].

While the use of targeted therapy described for CTLA4 haploinsufficiency seems very effective in reversing some of the manifestations of immune dysregulation, the possibility of viral reactivation during the treatment may be a limitation to the use of this drug for prolonged periods of time. For this reason, HSCT should be considered as a possible definitive therapy in patients with CTLA4 haploinsufficiency especially in case a fully matched donor is available. Results of HSCT are limited to a small cohort of patients but have been encouraging. The best candidates for HSCT may be patients with severe clinical manifestations like autoimmune hepatitis with nodular regenerative hyperplasia and a limited response to treatment with immunomodulatory drugs [21].

\section{Lipopolysaccharide-Responsive and Beige-Like Anchor} Deficiency

Lipopolysaccharide-responsive and beige-like anchor (LRBA) is a cytosolic protein that colocalizes with CTLA4 in endosomes; LRBA acts as a chaperone for CTLA4, enabling recycling of the molecule and the suppressive capacity of Treg cells. In the absence of LRBA, CTLA4 is targeted for lysosomal degradation, and its expression on Treg cells and activated conventional T cells is significantly diminished [22]. A deficiency of LRBA leads to an autosomal recessive form of CID that presents with hypogammaglobulinemia, a higher susceptibility to infections, and autoimmunity [23]. Since the original description, the clinical phenotype of the disease has broadened to include many more conditions of immune dysregulation like enteropathy, autoimmune blood cytopenias, and nonmalignant lymphoproliferation leading to splenomegaly and lymphadenopathy. Other autoimmune manifestations including autoimmune hepatitis, thyroid disease, diabetes type I, alopecia, myasthenia gravis, uveitis, and polyarthritis have also been described; Bratanic et al. [26] also reported multifocal gastric adenocarcinoma in a patient with LRBA deficiency [23-25, 27].

As enteropathy and polyendocrinopathy could coexist in the same patient, a phenotype that mimics the immune dysregulation, polyendocrinopathy, X-linked (IPEX) syndrome may be observed [28]. Respiratory complications including interstitial lung disease, granulomas, and bronchiectasis mainly related to viral and bacterial infections are also common $[25,27]$. Involvement of the cen- 
tral nervous system has been observed in one fourth of the patients and may manifest with demyelination but also with brain atrophy and granulomas [25].

The immunological phenotype of LRBA deficiency includes hypogammaglobulinemia, and a markedly reduced proportion of switched memory B cells along with an increase in double-negative T cells and circulating Tfh $[23,25,27,29]$. Most of the patients present with reduced numbers and impaired function of Treg cells [28] that express decreased levels of FOXP3, CD25, and CTLA4 proteins $[22,28]$.

The phenotypical similarities with CTL4 haploinsufficiency may be explained by the functional interplay between LRBA and CTLA4 proteins and have offered a rational basis to attempt treatment with abatacept also for LRBA deficiency [22]. Abatacept was shown to be effective in reversing lymphocytic interstitial lung disease and blood cytopenias in 3 LRBA patients; however, the enteropathy was not as responsive, and sirolimus and other immunosuppressant drugs needed to be added to control the disease. Regarding the immunological phenotype, treatment with abatacept was associated with an increased naive:effector T-cell ratio and improved antibody responses to polysaccharide vaccines. Moreover, circulating Tfh frequencies and a soluble IL-2R $\alpha$ chain have been shown to be useful markers to assess the clinical response of LRBA- and CTLA-4-deficient patients to CTLA4-Ig therapy [29]. Prolonged treatment over several years resulted in minimal infectious or autoimmune complications [22]. Moreover, in vitro studies with chloroquine, an inhibitor of lysosomal degradation, showed efficacy in preventing the loss of CTLA4 in patients with an LRBA deficiency [22], suggesting that both chloroquine and hydroxychloroquine may be used as immunomodulatory drugs in this disease. However, as in CTLA4 haploinsufficiency, HSCT is the only potentially definitive treatment for LRBA deficiency; only few patients have been transplanted so far and further evidence is needed to assess its safety and efficacy $[27,30]$.

\section{STAT1 and STAT3 GOF}

Signal transducer and activator of transcription (STAT) molecules account for 7 different proteins that are expressed by immune and nonimmune cells and play a key role in immune and inflammatory responses. Several cytokines and growth factors (type I, type II, type III interferons [IFN], IL-6, EGF, PDGF, IL-21, and IL-23) bind to their own receptor on the cell surface and recruit 4 different Janus kinases (JAK) to the receptor intracytoplasmic tail. Upon activation and phosphorylation, JAK phos-

Mechanism-Based Treatment of Primary Immunodeficiencies phorylate the intracellular tail of the cytokine receptor, offering a binding site for the Src homology 2 (SH2) domain of cytosolic STAT molecules that, upon phosphorylation, form homodimers or heterodimers and translocate to the nucleus to promote transcription of target genes that regulate various immune pathways and control cell proliferation, differentiation, survival, and death [31]. Although the protein functions of JAK and STAT proteins partially overlap in the transduction of cytokine-mediated signaling, mutations in individual JAK and STAT genes are associated with specific phenotypes [31].

The binding of type I and type II IFN, IL-10, IL-2, IL21 , and other cytokines to their cognate receptors activates STAT1. The signal is transmitted through JAK1, JAK2, and JAK3. These molecules act in different combinations [32]. Dupuis et al. [33] showed that biallelic LOF mutations of STAT1 lead to a severe susceptibility to viral and mycobacterial infections due to an impaired response to both type I and type II IFN. Later, the same group showed that heterozygous mutations with a dominant negative effect cause an increased susceptibility to mycobacterial disease [34]. Furthermore, heterozygous GOF mutations in STAT1 have been primarily associated with chronic mucocutaneous candidiasis (CMC) $[35,36]$. Most of these mutations occur in the coiled-coil and the DNA-binding domains of the protein $[35,37]$ leading to increased STAT1 expression, increased STAT1 phosphorylation, or delayed dephosphorylation after stimulation with IFN- $\alpha$, IFN- $\gamma$, and IL-27 $[38,39]$. The identification of many more patients with STAT1 GOF mutations broadened the phenotypical spectrum of the disease. Toubiana et al. [37] reported on a cohort of 274 patients collected from multiple centers. CMC was present in virtually all of the subjects in childhood, and $74 \%$ of the patients also suffered from bacterial infections, mainly affecting the respiratory tract and skin, with $S$. aureus being the most common bacterium isolated. Viral infections, especially due to Herpesviridae, and mycobacterial disease were also reported [37]. Severe and invasive fungal infection were due not only to Candida but included mucormycosis [40], coccidioidomycosis, histoplasmosis, and aspergillosis [41].

Autoimmunity and immune dysregulation were reported in 30\% of the patients [38]. Hypothyroidism, blood cytopenias, juvenile diabetes, and systemic lupus erythematosus are especially common, but inflammatory bowel disease, arthritis, and multiple sclerosis have also been reported [37]. In STAT1 GOF patients, all of these immune-related disorders are often refractory to conventional treatment and they are challenging to treat.

Med Princ Pract 2020;29:101-112 
Moreover, cerebral aneurysms and vasculopathy are frequently observed and put patients with STAT1 GOF mutations at risk for intracranial bleeding if not diagnosed in a timely fashion $[37,38]$. Malignancies (including squamous cell carcinoma) may also occur [37]. The immunological phenotype includes decreased numbers and function of T, B, and/or NK cells, and hypogammaglobulinemia [37]. In a small number of patients a loss of $\mathrm{B}$ cells over time has been reported. This immunological feature is associated with poor clinical conditions and worse outcomes (pers. obs.). Furthermore, patients have circulating Tfh cells that have an aberrant phenotype, with a reduced proportion of CCR6+ cells (effective Bhelper Tfh cells) and an increased expression of IFN- $\gamma$ and programmed death 1 (PD1) proteins [42].

HSCT is in the only curative treatment and it has been attempted in patients with complicated disease. However, low survival and increased rates of graft failure have been reported after HSCT in STAT1 GOF patients [37, 43, 44].

Conservative medical therapy is based on long-term use of systemic antifungal agents; however, this approach has not always been successful due to the side effects of medications and the onset of resistance. Antibacterial and antiviral medications, and the use of immunosuppressive drugs, are often needed as well. Despite these therapies, the rate of mortality by 60 years of age is more than $10 \%$ in patients without invasive infections, cancer, and/or symptomatic aneurysms, but it is around $70 \%$ in those with prominent immune dysregulation and autoimmunity.

The molecular understanding of the JAK-STAT pathway has paved the way for the use of targeted pharmacologic inhibitors in patients with STAT1 GOF [45-47]. Currently the following 5 different small molecule JAK inhibitors (Jakinibs) are available: tofacitinib (a JAK1 and JAK3 inhibitor), ruxolitinib (JAK1 and JAK2 inhibitor), baricitinib (JAK1 and JAK2 inhibitor), filgotinib (a more selective JAK1 inhibitor), and decernotinib (a selective JAK3 inhibitor) [48, 49]. The 5 different molecules display slightly different toxicity profiles and availabilities.

Recently, treatment with Jakinibs was assessed in 11 STAT1 GOF patients. Clinical features included severe fungal infections in 6 patients (including CMC in 5 patients and disseminated coccidiomycosis in 1 patient), cytopenias $(n=6)$, autoimmune enteropathy $(n=5)$, and hepatitis $(n=5)$. Polyendocrinopathy and interstitial lung disease were also present in some patients [45].

Therapy with Jakinibs led to improvement of cytopenias, interstitial lung disease, and total parenteral nutrition-dependent enteropathy. Moreover, improvements have been observed regarding the ability to control infections. CMC was responsive to the treatment [45] while the disseminated coccidiomycosis progressively worsened and the patient eventually died. Another case was described in the same cohort with severe fungal infection and unfavorable outcomes despite therapy with a Jakinib, suggesting that the treatment may be insufficient to reverse systemic fungal infection [45]. Meesilpavikkai et al. [47] reported on the efficacy of baricitinib in 1 patient who had a reduction of the IFN signature and downstream IFN activation.

Inhibition of STAT1 phosphorylation and improved NK-cell cytotoxicity have been observed when peripheral blood mononuclear cells from patients with STAT1 GOF are treated with Jakinibs in vitro $[50,51]$. Furthermore, normalization of the proportions of TH1, TH17, and Tfh cells has been associated with clinical improvement in patients undergoing treatment [46].

Considering the side effects, therapy with Jakinibs may cause elevation of liver enzymes and a mild decrease in the platelet count. Epstein-Barr virus, cytomegalovirus, BK, and JC viremia should always be monitored in patients on treatment, especially if more than one immunosuppressive agent is administered in combination with Jakinibs. Herpes zoster infections have been reported in 2 patients receiving this therapy, and prophylaxis with acyclovir or valacyclovir needs to be considered [45].

Type I, II, and III IFN and IL-6, IL-10, and IL-21 are the key molecules that activate STAT3 upon intracellular signaling. While dominant negative germline mutations in STAT3 result in an autosomal dominant hyper IgE (AD-HIES) syndrome characterized by eczema, skin abscesses, recurrent pneumonias leading to pneumatoceles, and skeletal and connective tissue abnormalities, the original description of heterozygous STAT3 GOF mutations included patients with early-onset autoimmunity, including type 1 diabetes in infancy [52]. Flanagan et al. [52] showed that transfection of mutant STAT3 into HEK293T cells led to augmented transcriptional activity compared to transfection of wild-type STAT3. More patients with STAT3 GOF mutations were identified shortly thereafter [53], expanding the clinical phenotype to nonmalignant lymphoproliferation including lymphadenopathy, splenomegaly and interstitial pneumonia and recurrent infections due to nontuberculous mycobacteria, fungi, and viruses. Acquired short stature is also a peculiar feature of the disease [53-55], while the most common autoimmune manifestations include enteropathy and cytopenias [53]. Studies on these families revealed that there are some genetically affected members with an 
absent or very mild phenotype consistent with incomplete penetrance and variable expressivity [56].

Regarding the immunological phenotype, these patients may present with $\mathrm{T}$-cell lymphopenia with a low number of Treg cells and skewing of CD4+ T cells to the $\mathrm{TH} 1$ phenotype, an increased proportion of double-negative TCR $\alpha \beta+\mathrm{T}$ cells, hypogammaglobulinemia with terminal B cell maturation arrest, and a decreased number of circulating dendritic cells, eosinophils, TH17 cells, and NK cells $[52,53,56]$. All of these immunological abnormalities are caused by enhanced transcriptional activity of STAT3 or delayed kinetics of STAT3 dephosphorylation. The profound immune dysregulation associated with STAT3 GOF mutations is consistent with the known role of STAT3 signaling in promoting inflammation and TH17 cell differentiation, and inhibiting Treg cells $[57,58]$.

Furthermore, STA T3 GOF mutations lead to a decrease in STAT1 and STAT5 phosphorylation [56]. In particular, decreased levels of phosphorylated STAT5 have been proposed as a possible mechanism leading to diminished growth and stature postnatally, reflecting impaired signaling through the growth hormone receptor [59].

Characterization of the molecular abnormalities underlying the disease offered the basis to treat these patient with an IL-6-targeted therapy. Treatment with tocilizum$\mathrm{ab}$ an anti-IL6 receptor (IL6R) mAb turned out to be effective in 1 patient with an STAT3 GOF mutation who had severe arthritis and scleroderma-like disease that did not respond to conventional immunosuppressant therapies; significant improvement of contractures and inflammatory markers and normalization of the proportion of TH17 cells have been reported [56]. Three additional patients did benefit from administration of tocilizumab to control autoimmune manifestations, including hepatitis and enteropathy, lymphoproliferation, and interstitial lung disease. However, this treatment was not sufficient to completely reverse the immune dysregulation, and Jakinibs had to be added as well. In 3 other patients, tocilizumab and a Jakinib were started simultaneously, leading to complete resolution of manifestations of immune dysregulation. These data suggest that the combination of IL- 6 blockade and Jakinib therapy is an effective treatment strategy, and both agents should be considered as combination therapy in the treatment of immune dysregulation in patients with STAT3 GOF mutations [45]. Finally, 2 patients with refractory autoimmunity and severe clinical manifestations underwent HSCT; 1 of them died with disseminated adenovirus infection post-transplant, while the other was reported to be alive and in remission [56].

Mechanism-Based Treatment of Primary Immunodeficiencies

\section{Precision Medicine in Autoinflammatory Disorders}

\section{Interferonopathies}

Hyperactivation of the type I IFN response is the molecular signature of the interferonopathies, a group of disorders exacerbated by multiple triggers, including damaged nucleic acid in the cytosol. DNA sequences are recognized by cyclic guanosine monophosphate adenosine monophosphate synthase, a sensor molecule that in turn activates the stimulator of IFN genes (STING) [60-62]. STING promotes the inflammatory response by activating either nuclear factor- $\mathrm{kB}(\mathrm{NF}-\kappa \mathrm{B})$ or IFN regulatory factor 3 [63-65].

STING-associated diseases include early-onset severe vasculopathy [66] and familial chilblain lupus [67]. Both diseases are caused by GOF mutations in TMEM173, the gene that encodes for the STING protein. Early-onset cold-induced blistering rash, elevated inflammatory markers, and fever are the most frequent clinical manifestations. Moreover, small vessel vasculopathy that can lead to necrosis of digits has been reported as a severe complication of this disease [66].

Augmented type I IFN signatures have also been associated with genetic defects in the components of the immunoproteasome, a complex proteolytic machinery derived from the constitutive proteasome and abundantly expressed in immune cells. The immunoproteasome plays a critical role in the immune system because it processes proteins for antigen presentation and regulates activation of the NF- $\kappa B$ pathway and management of oxidative stress $[68,69]$. Mutations in multiple genes encoding for immunoproteasome subunits, such as PSMA3, PSMB4, PSMB8, and PSMB9, have been linked to autoinflammatory syndromes. The clinical phenotype in this group of diseases is summarized by the acronym CANDLE, which stands for early-onset Chronic Atypical Neutrophilic Dermatosis, Lipodystrophy, and Elevated temperature. In addition, a similar but not completely overlapping phenotype has been associated with proteasome maturation protein $(P O M P)$ mutations. This gene encodes for a protein that is crucial for immunoproteasome assembly $[68,70]$. POMP mutations lead to a peculiar phenotype that is characterized by immune deficiency, neonatal-onset Sweet syndrome, and autoimmunity [68].

Several immunosuppressive therapies including corticosteroids, methotrexate, azathioprine, cyclophosphamide, 6-mercaptopurine, etanercept, infliximab, rituximab, and IL-1 antagonist have been used alone or in combination in patients with CANDLE and STING- associated diseases, but the response has been limited [66,

Med Princ Pract 2020;29:101-112

DOI: $10.1159 / 000503997$ 
$71,72]$. This is not surprising considering that stimulation with cGAMP of fibroblasts from patients with STING-associated diseases lead to increased transcription of IFNB1 but not of the genes encoding IL-1 or IL-6 [66]. Because of the upregulation of expression of type I IFN and activation of the JAK/STAT pathway, targeted therapy with Jakinibs has been implemented in order to neutralize the dysregulated IFN signature. In particular, treatment with baricitinib, tofacitinib, and ruxolitinib has been shown to decrease the severity of clinical manifestations and inflammatory markers in CANDLE syndrome and STING-associated vasculopathy [66, 67, 71-73]. Moreover, small-molecule directly targeting STING have been studied in a mouse model with promising results and may represent an alternative therapeutic strategy in the future [74]. A more traditional immunosuppressive approach with steroids, rapamycin, and rituximab has been helpful in limiting some of the clinical manifestations in POMP disease; however, based on recent findings of increased IFN signature in this disease, a more targeted approach could be considered in the future [68].

\section{Inflammasome Disorders}

Mounting adequate inflammatory responses against pathogens and molecules resulting from cellular damage is a key feature of the innate immune system. In this regard, rapid availability of proinflammatory cytokines including IL-1 and IL-18 represents a fundamental mechanism. Mutations in genes coding for inflammasome molecules have been associated with dysregulation in IL-1 production and signaling. In particular, mutations in NLRP3 and ILIRN genes lead to early-onset autoinflammatory diseases, cryopyrin-associated periodic syndromes (CAPS), and deficiency of IL-1 receptor antagonist.

NLRP3 is the most well-characterized cytoplasmic inflammasome sensor molecule. It is a protein of the NLR family and consists of a carboxy-terminal leucine-rich repeat (LRR) domain, a nucleotide-binding NACHT domain, and an amino-terminal PYRIN (PYD) domain. Upon stimulation, the NLRP3 inflammasome activates proteolytic enzymes that cleave the inactive pro-IL-1 $\beta$ and pro-IL-18 molecules into their active forms. In patients with CAPS, NLRP3 mutations lead to constitutive hyperactivation of the inflammasome [75]. These mutations result in GOF, as demonstrated by spontaneous inflammasome formation in the absence of activating signals [76,77]. More than 200 mutations in the NLRP3 gene associated with CAPS have been reported [78].
In 2001, NLRP3 mutations were first described as the cause of familial cold autoinflammatory syndrome [79] and Muckle-Wells syndrome [80]. Familial cold autoinflammatory syndrome is an early-onset disease with intermittent cold-induced neutrophilic urticaria, arthralgia, increased inflammatory markers, and fever. MuckleWells syndrome also manifests during infancy with painful and swollen joints, non-itchy rash, mild to moderate fever, and, in some cases, conjunctivitis. During adolescence, patients develop sensorineural hearing loss caused by progressive nerve damage. Progressive kidney disease due to amyloidosis is reported in about one third of patients affected by Muckle-Wells syndrome [80].

NLRP3 mutations were shown to be associated with neonatal onset multisystem inflammatory disease [76, 77]. Together with many features present in the other relatively milder forms of CAPS, patients with neonatal onset multisystem inflammatory disease often manifest central nervous system involvement, with presence of cerebral calcifications.

Biallelic LOF mutations of the IL1RN gene are responsible for the deficiency of IL-1 receptor antagonist (DIRA). These mutations lead to a reduced expression of an antagonist of IL-1 signaling and a consequent elevated production of IL-1 $\beta$. The disease shares some similarities with CAPS. The onset is typically neonatal, and patients suffer from fevers, rash, joint swelling, oral mucosal lesions, and bone abnormalities, including wide clavicles and ribs, periosteal elevation along the long bones, and osteolytic lesions [35, 81].

Considering the specific molecular pathway, trials with IL-1 antagonists has been performed. Anakinra, a recombinant IL-1 receptor antagonist with a short halflife, binds to the IL-1 receptor and thus impairs the binding of IL- $1 \alpha$ and IL-1 $\beta$. Rilonacept is a fusion protein in which IL-1R1 and IL-1RAcP are complexed to the Fc portion of IgG1 that binds IL- $1 \alpha$ and IL- $1 \beta$. Finally, canakinumab is a humanized $\operatorname{IgG} \mathrm{mAb}$ that selectively binds IL- $1 \beta$. Both rilonacept and canakinumab have longer half-lives compared to Anakinra.

Therapy with these newly discovered drugs was demonstrated to be effective in improving clinical manifestations, inflammatory markers, and the frequency of disease exacerbations in CAPS and DIRA [79, 82-84].

Another disease that has been successfully treated with a precision medicine approach using $\mathrm{mAb}$ against interleukins is the autoinflammatory syndrome due to NLRC4 (NLR family CARD domain-containing protein 4) GOF mutations. Macrophage activation syndrome and enterocolitis that present in the first few weeks of life represent 
the most frequent clinical manifestations [85-87]. The activation of NLRC4 protein causes the activation of both caspase- 1 and caspase- 8 and the consequent overproduction of proinflammatory cytokines, in particular IL-18 and IL-1 $\beta$ [85, 86, 88, 89]. While administration of IL-1 inhibitory molecules alone has been able to only partially modulate the clinical manifestations of the disease, treatment with a recombinant IL-18-binding protein (tadekinig- $\alpha$ ) was successful in a patient affected by NLRC4 GOF disease, and a phase 3 clinical trial (Clinical Trials No. NCT03113760) is currently enrolling patients [88]. In addition, a combination of rapamycin and IL- 1 inhibition has been attempted with the aim of reducing caspase- 1 activation and consequent overproduction of IL-1 and IL-18. Targeting of multiple disease-determining molecules was able to partially reverse clinical manifestations and should be considered in NLRC4 GOF patients whenever IL18 -binding protein is not easily available $[88,90]$.

\section{Other Examples of Precision Medicine in PID}

Escalating doses of IFN- $\alpha$ have been effective in patients with AR IFN- $\gamma$ R deficiency while IFN- $\gamma$ supplementation can be used in AD IFN- $\gamma$ R deficiency to decrease the burden of mycobacterial disease [91]. IFN- $\gamma$ may also be administered to patients with chronic granulomatous disease to aid the clearance of infectious agents [91]. Emapalumab, a human mAb inhibitor of IFN- $\gamma$, is an important option for the treatment of hemophagocytic lymphohistiocytosis in adult and pediatric patients. Alemtuzumab (anti-CD52) has also been effective in improving outcomes of hemophagocytic lymphohistiocytosis [91].
Ustekinumab, an $\mathrm{mAb}$ that binds to the $\mathrm{p} 40$ subunit common to IL-12 and IL-23, inhibiting IL-12 and IL-23 signaling and therefore downstream IL-17 response, has been effective in treating inflammatory complications of leukocyte adhesion deficiency type 1 including IBD and periodontitis [91]. Plerixafor is an inhibitor of the binding of CXCR4 to CXCL12 used as a therapeutic agent to counterbalance the increased cellular response to CXCL12 that characterizes WHIM syndrome [92]. TNF- $\alpha$ inhibition improved arthritis in patients with Blau syndrome due to mutations in the NOD2 gene [91].

\section{Conclusion}

The recent identification of many new PID and the characterization of their underlying molecular mechanisms has given clinical immunologists the opportunity to use novel therapeutic tools to modulate the immune system. Small molecules and biologics have shown to be particularly useful in patients with clinical manifestations of immune dysregulation and hyperinflammation. This precision medicine approach is effective in directly reversing disease-related symptoms and also in ameliorating the clinical condition of patients prior to HSCT.

\section{Funding Source}

This work was supported by the Division of Intramural Research, National Institutes of Allergy and Infectious Diseases (NIAID), National Institutes of Health (NIH), Bethesda, MD, USA.

\section{References}

1 Lucas CL, Kuehn HS, Zhao F, Niemela JE, Deenick EK, Palendira U, et al. Dominantactivating germline mutations in the gene en-

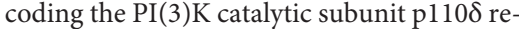
sult in T cell senescence and human immunodeficiency. Nat Immunol. 2014 Jan;15(1): 88-97.

2 Angulo I, Vadas O, Garçon F, Banham-Hall E, Plagnol V, Leahy TR, et al. Phosphoinositide 3 -kinase $\delta$ gene mutation predisposes to respiratory infection and airway damage. Science. 2013 Nov;342(6160):866-71.

3 Lucas CL, Zhang Y, Venida A, Wang Y, Hughes J, McElwee J, et al. Heterozygous splice mutation in PIK3R1 causes human immunodeficiency with lymphoproliferation due to dominant activation of PI3K. J Exp Med. 2014 Dec;211(13):2537-47.
4 Olbrich P, Lorenz M, Cura Daball P, Lucena JM, Rensing-Ehl A, Sanchez B, et al. Activated PI3K $\delta$ syndrome type 2: two patients, a novel mutation, and review of the literature. Pediatr Allergy Immunol. 2016 Sep;27(6): 640-4.

5 Petrovski S, Parrott RE, Roberts JL, Huang H, Yang J, Gorentla B, et al. Dominant splice site mutations in PIK3R1 cause Hyper IgM syndrome, lymphadenopathy and short stature. J Clin Immunol. 2016 Jul;36(5): 462-71.

6 Coulter TI, Chandra A, Bacon CM, Babar J, Curtis J, Screaton $\mathrm{N}$ et al.: Clinical spectrum and features of activated phosphoinositide 3-kinase delta syndrome: A large patient cohort study. J Allergy Clin Immunol. 2017;139: 597-606.
7 Elkaim E, Neven B, Bruneau J, Mitsui-Sekinaka K, Stanislas A, Heurtier Let al: Clinical and immunologic phenotype associated with activated phosphoinositide 3-kinase delta syndrome 2: A cohort study. J Allergy Clin Immunol. 2016;138:210-8.

8 Condliffe AM, Chandra A. Respiratory manifestations of the activated phosphoinositide 3-kinase delta syndrome. Front Immunol. 2018 Mar;9:338.

9 Maccari ME, Abolhassani H, Aghamohammadi A, Aiuti A, Aleinikova O, Bangs C, et al. Disease evolution and response to rapamycin in activated phosphoinositide 3-kinase delta syndrome: The European Society for Immunodeficiencies-Activated Phosphoinositide 3-Kinase delta Syndrome Registry. Front Immunol. 2018 Mar;9:543. 
10 Nademi Z, Slatter MA, Dvorak CC, Neven B, Fischer A, Suarez F, et al. Hematopoietic stem cell transplant in patients with activated PI3K delta syndrome. J Allergy Clin Immunol. 2017 Mar;139(3):1046-9.

11 Okano T, Imai K, Tsujita Y, Mitsuiki N, Yoshida K, Kamae C, et al. Hematopoietic stem cell transplantation for progressive combined immunodeficiency and lymphoproliferation in patients with activated phosphatidylinositol-3-OH kinase $\delta$ syndrome type 1. J Allergy Clin Immunol. 2019 Jan;143(1):266-75.

12 Rao VK, Webster S, Dalm VA, Šedivá A, van Hagen PM, Holland S, et al. Effective "activated $\mathrm{PI} 3 \mathrm{~K} \delta$ syndrome"-targeted therapy with the PI3K $\delta$ inhibitor leniolisib. Blood. 2017 Nov;130(21):2307-16.

13 Compagno M, Wang Q, Pighi C, Cheong TC, Meng FL, Poggio T, et al. Phosphatidylinositol 3-kinase $\delta$ blockade increases genomic instability in B cells. Nature. 2017 Feb; 542(7642):489-93.

14 Krummel MF, Allison JP. Pillars article: CD28 and CTLA- 4 have opposing effects on the response of $\mathrm{T}$ cells to stimulation. J Immunol. 2011;187:3459-65.

15 Kuehn HS, Ouyang W, Lo B, Deenick EK, Niemela JE, Avery DT, et al. Immune dysregulation in human subjects with heterozygous germline mutations in CTLA4. Science. 2014 Sep;345(6204):1623-7.

16 Schubert D, Bode C, Kenefeck R, Hou TZ, Wing JB, Kennedy A, et al. Autosomal dominant immune dysregulation syndrome in humans with CTLA4 mutations. Nat Med. 2014 Dec;20(12):1410-6.

17 Schwab C, Gabrysch A, Olbrich P, Patiño V, Warnatz K, Wolff D, et al. Phenotype, penetrance, and treatment of 133 cytotoxic T-lymphocyte antigen 4-insufficient subjects. J Allergy Clin Immunol. 2018 Dec;142(6):193246.

18 Herrero-Beaumont G, Martínez Calatrava MJ, Castañeda S. Abatacept mechanism of action: concordance with its clinical profile. Reumatol Clin. 2012 Mar-Apr;8(2):78-83.

19 Lee S, Moon JS, Lee CR, Kim HE, Baek SM, Hwang S, et al. Abatacept alleviates severe autoimmune symptoms in a patient carrying a de novo variant in CTLA-4. J Allergy Clin Immunol. 2016 Jan;137(1):327-30.

20 Navarini AA, Hruz P, Berger CT, Hou TZ, Schwab C, Gabrysch A et al: Vedolizumab as a successful treatment of CTLA-4-associated autoimmune enterocolitis. J Allergy Clin Immunol. 2017;139:1043-6.

21 Slatter MA Engelhardt KR, Burroughs LM, Arkwright PD, Nademi Z, Skoda-Smith S, et al.: Hematopoietic stem cell transplantation for CTLA4 deficiency. J Allergy Clin Immunol. 2016;138:615-9.

22 Lo B, Zhang K, Lu W, Zheng L, Zhang Q, Kanellopoulou C, et al. AUTOIMMUNE DISEASE. Patients with LRBA deficiency show CTLA4 loss and immune dysregulation responsive to abatacept therapy. Science. 2015 Jul;349(6246):436-40.
23 Lopez-Herrera G, Tampella G, Pan-Hammarström Q, Herholz P, Trujillo-Vargas CM, Phadwal K, et al. Deleterious mutations in LRBA are associated with a syndrome of immune deficiency and autoimmunity. Am J Hum Genet. 2012 Jun;90(6):986-1001.

24 Lévy E, Stolzenberg MC, Bruneau J, Breton S, Neven B, Sauvion S, et al. LRBA deficiency with autoimmunity and early onset chronic erosive polyarthritis. Clin Immunol. 2016 Jul; 168:88-93.

25 Alkhairy OK, Abolhassani H, Rezaei N, Fang $\mathrm{M}$, Andersen KK, Chavoshzadeh $\mathrm{Z}$, et al. Spectrum of phenotypes associated with mutations in LRBA. J Clin Immunol. 2016 Jan; 36(1):33-45.

26 Bratanič N, Kovač J, Pohar K, Trebušak Podkrajšek K, Ihan A, Battelino T, et al. Multifocal gastric adenocarcinoma in a patient with LRBA deficiency. Orphanet J Rare Dis. 2017 Jul;12(1):131.

27 Gámez-Díaz L, August D, Stepensky P, RevelVilk S, Seidel MG, Noriko M, et al. The extended phenotype of LPS-responsive beigelike anchor protein (LRBA) deficiency. $\mathrm{J} \mathrm{Al}-$ lergy Clin Immunol. 2016 Jan;137(1):223-30.

28 Charbonnier LM, Janssen E, Chou J, Ohsumi TK, Keles S, Hsu JT, et al. Regulatory T-cell deficiency and immune dysregulation, polyendocrinopathy, enteropathy, X-linked-like disorder caused by loss-of-function mutations in LRBA. J Allergy Clin Immunol. 2015 Jan;135(1):217-27.

29 Alroqi FJ, Charbonnier LM, Baris S, Kiykim A, Chou J, Platt CD, et al. Exaggerated follicular helper T-cell responses in patients with LRBA deficiency caused by failure of CTLA4mediated regulation. J Allergy Clin Immunol. 2018 Mar;141(3):1050-1059.e10.

30 Seidel MG, Böhm K, Dogu F, Worth A, Thrasher A, Florkin B, et al.: Long-term remission after allogeneic hematopoietic stem cell transplantation in LPS-responsive beigelike anchor (LRBA) deficiency. J Allergy Clin Immunol. 2015;135:1384-90.

31 Casanova JL, Holland SM, Notarangelo LD. Inborn errors of human JAKs and STATs. Immunity. 2012 Apr;36(4):515-28.

32 O'Shea JJ, Plenge R. JAK and STAT signaling molecules in immunoregulation and immune-mediated disease. Immunity. 2012 Apr;36(4):542-50.

33 Dupuis S, Jouanguy E, Al-Hajjar S, Fieschi C, Al-Mohsen IZ, Al-Jumaah S, et al. Impaired response to interferon-alpha/beta and lethal viral disease in human STAT1 deficiency. Nat Genet. 2003 Mar;33(3):388-91.

34 Dupuis S, Dargemont C, Fieschi C, Thomas$\sin \mathrm{N}$, Rosenzweig S, Harris J, et al. Impairment of mycobacterial but not viral immunity by a germline human STAT1 mutation. Science. 2001 Jul;293(5528):300-3.

35 Liu L, Okada S, Kong XF, Kreins AY, Cypowyj S, Abhyankar A, et al. Gain-of-function human STAT1 mutations impair IL-17 immunity and underlie chronic mucocutaneous candidiasis. J Exp Med. 2011 Aug;208(8):1635-48.
36 van de Veerdonk FL, Plantinga TS, Hoischen A, Smeekens SP, Joosten LA, Gilissen C, et al. STAT1 mutations in autosomal dominant chronic mucocutaneous candidiasis. N Engl J Med. 2011 Jul;365(1):54-61.

37 Toubiana J, Okada S, Hiller J, Oleastro M, Lagos Gomez M, Aldave Becerra JC, et al.; International STAT1 Gain-of-Function Study Group. Heterozygous STAT1 gain-of-function mutations underlie an unexpectedly broad clinical phenotype. Blood. 2016 Jun; 127(25):3154-64.

38 Uzel G, Sampaio EP, Lawrence MG, Hsu AP, Hackett M, Dorsey MJ, et al. Dominant gainof-function STAT1 mutations in FOXP3 wild-type immune dysregulation-polyendocrinopathy-enteropathy-X-linked-like syndrome. J Allergy Clin Immunol. 2013 Jun; 131(6):1611-23.

39 Depner M, Fuchs S, Raabe J, Frede N, Glocker C, Doffinger R, et al. The extended clinical phenotype of 26 patients with chronic mucocutaneous candidiasis due to gain-of-function mutations in STAT1. J Clin Immunol. 2016 Jan;36(1):73-84.

40 Kumar N, Hanks ME, Chandrasekaran P, Davis BC, Hsu AP, Van Wagoner NJ, et al. Gain-of-function signal transducer and activator of transcription 1 (STAT1) mutation-related primary immunodeficiency is associated with disseminated mucormycosis. J Allergy Clin Immunol. 2014 Jul;134(1): 236-9.

41 Sampaio EP, Hsu AP, Pechacek J, Bax HI, Dias DL, Paulson ML, et al. Signal transducer and activator of transcription 1 (STAT1) gain-of-function mutations and disseminated coccidioidomycosis and histoplasmosis. J Allergy Clin Immunol. 2013 Jun;131(6):162434.

42 Ma CS,Wong N, Rao G, Avery DT, Torpy J, Hambridge T, et al.: Monogenic mutations differentially affect the quantity and quality of $\mathrm{T}$ follicular helper cells in patients with human primary immunodeficiencies. J Allergy Clin Immunol. 2015;136:993-1006.

43 Leiding JW, Okada S, Hagin D, Abinun M, Shcherbina A, Balashov DN, et al.: Hematopoietic stem cell transplantation in patients with gain-of-function signal transducer and activator of transcription $1 \mathrm{mu}$ tations. J Allergy Clin Immunol. 2018;141: 704-17.

44 Aldave JC, Cachay E, Núñez L, Chunga A, Murillo S, Cypowyj S, et al. A 1-year-old girl with a gain-of-function STAT1 mutation treated with hematopoietic stem cell transplantation. J Clin Immunol. 2013 Nov;33(8): 1273-5.

45 Forbes LR, Vogel TP, Cooper MA, CastroWagner J, Schussler E, Weinacht KG, et al. Jakinibs for the treatment of immune dysregulation in patients with gain-of-function signal transducer and activator of transcription 1 (STAT1) or STAT3 mutations. J Allergy Clin Immunol. 2018 Nov;142(5): 1665-9. 
46 Higgins E, Al Shehri T, McAleer MA, Conlon $\mathrm{N}$, Feighery C, Lilic D, et al. Use of ruxolitinib to successfully treat chronic mucocutaneous candidiasis caused by gain-of-function signal transducer and activator of transcription 1 (STAT1) mutation. J Allergy Clin Immunol. 2015 Feb;135(2):551-3.

47 Meesilpavikkai K, Dik WA, Schrijver B, Nagtzaam NMA, Posthumus-van Sluijs SJ, van Hagen PM, et al.: Baricitinib treatment in a patient with a gain-of-function mutation in signal transducer and activator of transcription 1 (STAT1). J Allergy Clin Immunol. 2018;142:328-30

48 Hirahara K, Schwartz D, Gadina M, Kanno Y, O'Shea JJ. Targeting cytokine signaling in autoimmunity: back to the future and beyond. Curr Opin Immunol. 2016 Dec;43:89-97.

49 Roskoski R Jr. Janus kinase (JAK) inhibitors in the treatment of inflammatory and neoplastic diseases. Pharmacol Res. 2016 Sep;111: 784-803.

50 Vargas-Hernandez A, Mace EM, Zimmerman O, Zerbe CS, Freeman AF, Rosenzweig S et al: Ruxolitinib partially reverses functional natural killer cell deficiency in patients with signal transducer and activator of transcription 1 (STAT1) gain-of-function mutations. J Allergy Clin Immunol. 2018;141:2142-55.

51 Weinacht KG, Charbonnier LM, Alroqi F, Plant A, Qiao Q, Wu H, et al.: Ruxolitinib reverses dysregulated $\mathrm{T}$ helper cell responses and controls autoimmunity caused by a novel signal transducer and activator of transcription 1 (STAT1) gain-of-function mutation. J Allergy Clin Immunol. 2017;139:1629-40.

52 Flanagan SE, Haapaniemi E, Russell MA, Caswell R, Allen HL, De Franco E, et al. Activating germline mutations in STAT3 cause earlyonset multi-organ autoimmune disease. Nat Genet. 2014 Aug;46(8):812-4.

53 Haapaniemi EM, Kaustio M, Rajala HL, van Adrichem AJ, Kainulainen L, Glumoff V, et al. Autoimmunity, hypogammaglobulinemia, lymphoproliferation, and mycobacterial disease in patients with activating mutations in STAT3. Blood. 2015 Jan;125(4):639-48.

54 Milner JD, Brenchley JM, Laurence A, Freeman AF, Hill BJ, Elias KM, et al. Impaired $\mathrm{T}(\mathrm{H}) 17$ cell differentiation in subjects with autosomal dominant hyper-IgE syndrome. Nature. 2008 Apr;452(7188):773-6.

55 Haddad E. STAT3: too much may be worse than not enough! Blood. 2015 Jan;125(4): 583-4.

56 Milner JD, Vogel TP, Forbes L, Ma CA, Stray-Pedersen A, Niemela JE, et al. Earlyonset lymphoproliferation and autoimmunity caused by germline STAT3 gain-of-function mutations. Blood. 2015 Jan;125(4):591-9.

57 Camporeale A, Poli V. IL-6, IL-17 and STAT3: a holy trinity in auto-immunity? Front Biosci. 2012 Jun; 17(7):2306-26.

58 Kane A, Deenick EK, Ma CS, Cook MC, Uzel G, Tangye SG. STAT3 is a central regulator of lymphocyte differentiation and function. Curr Opin Immunol. 2014 Jun;28:49-57.
59 Palmer DC, Restifo NP. Suppressors of cytokine signaling (SOCS) in T cell differentiation, maturation, and function. Trends Immunol. 2009 Dec;30(12):592-602.

60 Burdette DL, Vance RE. STING and the innate immune response to nucleic acids in the cytosol. Nat Immunol. 2013 Jan;14(1):19-26.

61 Diner EJ, Burdette DL, Wilson SC, Monroe KM, Kellenberger CA, Hyodo M, et al. The innate immune DNA sensor cGAS produces a noncanonical cyclic dinucleotide that activates human STING. Cell Rep. 2013 May; 3(5):1355-61.

62 Mackenzie KJ, Carroll P, Lettice L, Tarnauskaite Ž, Reddy K, Dix F, et al. Ribonuclease $\mathrm{H} 2$ mutations induce a cGAS/ STING-dependent innate immune response. EMBO J. 2016 Apr;35(8):831-44.

63 Abe T, Barber GN. Cytosolic-DNA-mediated, STING-dependent proinflammatory gene induction necessitates canonical NF- $\mathrm{kB}$ activation through TBK1. J Virol. 2014 May; 88(10):5328-41.

64 Takaoka A, Taniguchi T. Cytosolic DNA recognition for triggering innate immune responses. Adv Drug Deliv Rev. 2008 Apr;60(7): 847-57.

65 Takaoka A, Wang Z, Choi MK, Yanai H, Negishi H, Ban T, et al. DAI (DLM-1/ZBP1) is a cytosolic DNA sensor and an activator of innate immune response. Nature. 2007 Jul; 448(7152):501-5.

66 Liu Y, Jesus AA, Marrero B, Yang D, Ramsey SE, Sanchez GA, et al. Activated STING in a vascular and pulmonary syndrome. $\mathrm{N}$ Engl J Med. 2014 Aug;371(6):507-18.

67 König N, Fiehn C, Wolf C, Schuster M, Cura Costa E, Tüngler V, et al. Familial chilblain lupus due to a gain-of-function mutation in STING. Ann Rheum Dis. 2017 Feb;76(2):468-72.

68 Poli MC, Ebstein F, Nicholas SK, de Guzman MM, Forbes LR, Chinn IK, et al.; Undiagnosed Diseases Network members. Heterozygous truncating variants in POMP escape nonsense-mediated decay and cause a unique immune dysregulatory syndrome. Am J Hum Genet. 2018 Jun;102(6):1126-42.

69 Budenholzer L, Cheng CL, Li Y, Hochstrasser M. Proteasome Structure and Assembly. J Mol Biol. 2017 Nov;429(22):3500-24.

70 Manthiram K, Zhou Q, Aksentijevich I, Kastner DL. The monogenic autoinflammatory diseases define new pathways in human innate immunity and inflammation. Nat Immunol. 2017 Jul;18(8):832-42.

71 Kim H, Sanchez GA, Goldbach-Mansky R. Insights from Mendelian Interferonopathies: Comparison of CANDLE, SAVI with AGS, Monogenic Lupus. J Mol Med (Berl). 2016 Oct;94(10):1111-27.

72 Liu Y, Ramot Y, Torrelo A, Paller AS, Si N, Babay S, et al. Mutations in proteasome subunit $\beta$ type 8 cause chronic atypical neutrophilic dermatosis with lipodystrophy and elevated temperature with evidence of genetic and phenotypic heterogeneity. Arthritis Rheum. 2012 Mar;64(3):895-907.
73 Sanchez GA, Reinhardt A, Ramsey S, Wittkowski H, Hashkes PJ, Berkun Y, et al. JAK1/2 inhibition with baricitinib in the treatment of autoinflammatory interferonopathies. J Clin Invest. 2018 Jul;128(7):3041-52.

74 Haag SM, Gulen MF, Reymond L, Gibelin A, Abrami L, Decout A, et al. Targeting STING with covalent small-molecule inhibitors. Nature. 2018 Jul;559(7713):26973.

75 Martinon F, Burns K, Tschopp J. The inflammasome: a molecular platform triggering activation of inflammatory caspases and processing of proIL-beta. Mol Cell. 2002 Aug; 10(2):417-26.

76 Broderick L, De Nardo D, Franklin BS, Hoffman HM, Latz E. The inflammasomes and autoinflammatory syndromes. Annu Rev Pathol. 2015;10(1):395-424.

77 Feldmann J, Prieur AM, Quartier P, Berquin $\mathrm{P}$, Certain S, Cortis E, et al. Chronic infantile neurological cutaneous and articular syndrome is caused by mutations in CIAS1, a gene highly expressed in polymorphonuclear cells and chondrocytes. Am J Hum Genet. 2002 Jul;71(1):198-203.

78 Sarrauste de Menthière $C$, Terrière $S$, Pugnère D, Ruiz M, Demaille J, Touitou I. INFEVERS: the Registry for FMF and hereditary inflammatory disorders mutations. Nucleic Acids Res. 2003 Jan;31(1):282-5.

79 Hoffman HM, Mueller JL, Broide DH, Wanderer AA, Kolodner RD. Mutation of a new gene encoding a putative pyrin-like protein causes familial cold autoinflammatory syndrome and Muckle-Wells syndrome. Nat Genet. 2001 Nov;29(3):301-5.

80 Cuisset L, Drenth JP, Berthelot JM, Meyrier A, Vaudour G, Watts RA, et al. Genetic linkage of the Muckle-Wells syndrome to chromosome 1q44. Am J Hum Genet. 1999 Oct; 65(4):1054-9.

81 Aksentijevich I, Masters SL, Ferguson PJ, Dancey P, Frenkel J, van Royen-Kerkhoff A, et al. An autoinflammatory disease with deficiency of the interleukin-1-receptor antagonist. N Engl J Med. 2009 Jun;360(23):242637.

82 Goldbach-Mansky R, Dailey NJ, Canna SW, Gelabert A, Jones J, Rubin BI, et al. Neonatal-onset multisystem inflammatory disease responsive to interleukin-1beta inhibition. N Engl J Med. 2006 Aug;355(6): 581-92.

83 Hoffman HM, Rosengren S, Boyle DL, Cho JY, Nayar J, Mueller JL, et al. Prevention of cold-associated acute inflammation in familial cold autoinflammatory syndrome by interleukin-1 receptor antagonist. Lancet. 2004 Nov;364(9447):1779-85.

84 Hoffman HM, Throne ML, Amar NJ, Sebai M, Kivitz AJ, Kavanaugh A, et al. Efficacy and safety of rilonacept (interleukin-1 Trap) in patients with cryopyrin-associated periodic syndromes: results from two sequential placebo-controlled studies. Arthritis Rheum. 2008 Aug;58(8):2443-52. 
85 Romberg N, Al Moussawi K, Nelson-Williams C, Stiegler AL, Loring E, Choi M, et al. Mutation of NLRC4 causes a syndrome of enterocolitis and autoinflammation. Nat Genet. 2014 Oct;46(10):1135-9.

86 Romberg N, Vogel TP, Canna SW. NLRC4 inflammasomopathies. Curr Opin Allergy Clin Immunol. 2017 Dec;17(6):398-404.

87 Canna SW, de Jesus AA, Gouni S, Brooks SR, Marrero B, Liu Y, et al. An activating NLRC4 inflammasome mutation causes autoinflammation with recurrent macrophage activation syndrome. Nat Genet. 2014 Oct;46(10):11406.
88 Canna SW, Girard C, Malle L, de Jesus A, Romberg N, Kelsen J, et al. Life-threatening NLRC4associated hyperinflammation successfully treated with IL-18 inhibition. J Allergy Clin Immunol. 2017 May;139(5):1698-701.

89 Rauch I, Deets KA, Ji DX, von Moltke J, Tenthorey JL, Lee AY, et al. NAIP-NLRC4 inflammasomes coordinate intestinal epithelial cell expulsion with eicosanoid and IL-18 release via activation of caspase-1 and -8. Immunity. 2017 Apr;46(4):649-59.

90 Barsalou J, Blincoe A, Fernandez I, Dal-Soglio D, Marchitto L, Selleri S, et al. Rapamycin as an adjunctive therapy for NLRC4 associated macrophage activation syndrome. Front Immunol. 2018 Sep;9:2162.

91 Leiding JW, Forbes LR. Mechanism-based precision therapy for the treatment of primary immunodeficiency and primary immunodysregulatory diseases. J Allergy Clin Immunol Pract. 2019 Mar;7(3):761-73.

92 Notarangelo LD, Fleisher TA. Targeted strategies directed at the molecular defect: toward precision medicine for select primary immunodeficiency disorders. J Allergy Clin Immunol. 2017 Mar;139(3):715-23. 